\title{
Innovación abierta y rendimiento financiero en la industria manufacturera de México
}

\author{
García Vidales, Marisela Yazmín ${ }^{1}$ \\ Maldonado Guzmán, Gonzalo² \\ Alvarado Carrillo, Araceli ${ }^{2}$
}

\section{Resumen}

La presente investigación tiene como objetivo analizar la influencia que tiene la innovación abierta en el desempeño financiero de la industria manufacturera de México, para ello se realizó un estudio empírico con una muestra de 300 pequeñas y medianas empresas con un enfoque cuantitativo a través de la aplicación de un modelo de ecuaciones estructurales, el cual es uno de los métodos más adecuados para el análisis de dependencia. Si bien es cierto, que la innovación abierta es un constructo relativamente reciente en la actual literatura del marketing, también es cierto que es considerada por diversos investigadores, académicos y profesionales del campo de la innovación, como la estrategia empresarial que genera un mayor nivel de rendimiento financiero a las empresas que la han implementado. Los resultados obtenidos en este estudio empírico muestran que la innovación abierta ejerce un efecto positivo significativo en el rendimiento financiero de las empresas de la industria manufacturera de México, por lo cual es posible concluir, que las empresas que adopten la innovación abierta, tendrán mayores posibilidades de lograr un incremento significativo en su rendimiento financiero.

Palabras clave: innovación; innovación abierta; rendimiento financiero; rendimiento del negocio.

Recibido: 20.03.19 Aceptado: 20.06.19

Estudiante de doctorado en la Universidad Autónoma de Aguascalientes. E-mail: marisela_ vidales@hotmail.com

2 Profesor investigador de la Universidad Autónoma de Aguascalientes. E-mail:gmaldona@ correo.uaa.mx 


\title{
Open innovation and financial performance in Mexican manufacturing industry
}

\begin{abstract}
The objective of this research is to analyze the influence that open innovation has on the financial performance of the manufacturing industry in Mexico, for which, an empirical study was carried out using a sample of 300 small and medium enterprises with a quantitative approach through the application of a structural equation model, which is one of the most suitable methods for the analysis of dependence. In addition, while it is true that open innovation is a relatively recent construct in the current literature of marketing, it is also true that it is considered by several researchers, academics and professionals in the innovation field, as the business strategy that generates a higher level of financial performance to the companies that have implemented it. The obtained results in this empirical study show that open innovation has a significant positive effect on the financial performance of companies in the manufacturing industry in Mexico, so it is possible to conclude that firms that adopt open innovation will have greater possibilities of achieve a significant increase in their financial performance.
\end{abstract}

Keywords: Innovation, Open Innovation, Financial Performance, Business Performance.

\section{Introducción}

Actualmente, diversas organizaciones, particularmente las pequeñas empresas, están experimentando una apertura de sus actividades de innovación caracterizada por un incremento sustantivo en la colaboración con otras empresas para la obtención de información externa y el desarrollo de nuevos productos, servicios o procesos (Caputo et al, 2016). Por ello, la innovación abierta está emergiendo en las empresas como una actividad primordial, ya que básicamente la innovación abierta no solamente representa una parte esencial del actual modelo de los negocios, sino también genera diversas condiciones en la forma de hacer negocios tales como la reducción del ciclo de vida de los pro- ductos, la intensificación de la competencia global y la reducción de los costos de investigación y desarrollo (Caputo et al, 2016).

En este sentido, la relación entre la innovación abierta y el rendimiento financiero de las empresas está emergiendo en la literatura del campo del marketing como una primordial área de interés de estudio (Schroll y Mild, 2012). Asimismo, debido a la complejidad y heterogeneidad del término mismo de la innovación abierta, no es nada sencillo analizar e investigar los efectos que ésta tiene en el rendimiento financiero de las empresas (Ahn et al, 2015). Por lo tanto, aun cuando existe evidencia teórica que demuestra que la innovación abierta genera diversos beneficios para las organizaciones, primordialmente para las pe- 
queñas empresas, los resultados de los estudios empíricos publicados indican la existencia de contradicciones (Caputo et al, 2016), por un lado, la mayoría de los estudios han encontrado efectos positivos significativos en el rendimiento financiero y, por otro lado, también existen resultados negativos (Greco et al, 2015).

Sin embargo, la adquisición de conocimiento externo [inbound open innovation] ha recibido una mayor atención de investigadores, académicos y profesionales del campo del marketing que la explotación del conocimiento interno [outbound open innovation] (West y Bogers, 2014) por lo cual, consecuentemente, la relación existente entre la innovación abierta y el rendimiento financiero ha recibido una insuficiente atención (Lichtenthaler, 2015), de ahí la importancia de este estudio empírico. Además, empresas de distintas industrias y sectores están adoptado cada vez más la innovación abierta como parte de sus estrategias empresariales (Gambardella et al, 2007), ya que la innovación abierta permite a las empresas interactuar con otras empresas de su mismo o distinto sector, de tal manera que les permita explotar de manera conjunta los recursos tecnológicos y los canales de comercialización (Cooper, 2008).

Así, para mejorar significativamente el rendimiento financiero de las empresas una de las mejores decisiones estratégicas, es la adopción e implementación de la innovación abierta, ya que ello facilita no solamente la generación y/o adquisición de nuevo conocimiento (Chesbrough, 2006), sino también un crecimiento sostenido (Caputo et al, 2016), para lo cual las organizaciones, particularmente las pequeñas empresas, deberán contar con la tecnología necesaria que les permita aprovechar al máximo las bondades internas y externas que ofrece la innovación abierta (Vanhaverbeke et al, 2002). Por lo tanto, las empresas podrán tomar la decisión de la comercialización o no del licenciamiento de su tecnología basado en el posicionamiento que tengan en el mercado (Chesbrough, 2006), además de que la innovación abierta genera diversos beneficios cuando ésta forma parte de la estrategia empresarial (Fosfuri, 2006).

A pesar de la evidencia existente que demuestra los efectos positivos de la innovación abierta en el rendimiento financiero de las empresas, son relativamente pocos los estudios empíricos que analizan estos dos importantes tópicos, especialmente cuando se utilizan muestras con grandes cantidades de empresas (Lichtenthaler, 2008). Asimismo, un elevado porcentaje de los estudios publicados en la actual literatura, han analizado la innovación abierta desde un punto de vista teórico basado en estudios de casos, o se han enfocado en el análisis de una parte específica de la innovación abierta (West et al, 2006). La falta de evidencia empírica de los efectos que ejerce la innovación abierta en el rendimiento empresarial en general, dificulta demasiado la comparación de las ventajas y desventajas de la innovación abierta en las empresas (Caputo et al, 2016)

Bajo esta perspectiva, dado que son relativamente pocos los estudios empíricos publicados en la actual literatura del marketing que se han orientado en el análisis y discusión de la relación existente entre la innovación abierta y el rendimiento financiero en general, la principal contribución de este estudio empírico es el análisis y discusión de los efectos que ejerce la innovación abierta de las pequeñas empresas de 
un país de economía emergente, como es el caso de México, en el rendimiento financiero tal y como lo recomiendan Lichtenthaler, (2008, 2009), West et al, (2006) y Caputo et al, (2016).

\section{Contextualización de la rela- ción entre innovación abierta y rendimiento financiero}

Enlaúltimadécadadelactualmilenio la innovación abierta ha ganado mucha atención por diversos investigadores, académicos y profesionales del campo del marketing (Linstone, 2010). Además, la adquisición de conocimiento externo y las habilidades para la explotación del desarrollo tecnológico interno en las organizaciones, no es nada nuevo (Mowery, 2009). El término de innovación abierta generalmente difiere entre los investigadores y académicos, ya que algunos de ellos indican actividades similares utilizando distintos conceptos, tales como por ejemplo activos complementarios (Teece, 1986), capacidad de absorción (Cohen y Levinthal, 1990) y exploración y explotación (March, 1991), cambiando prácticamente estas actividades a partir de la publicación del trabajo de Chesbrough (2003a), a partir del cual inició la proliferación el concepto de innovación abierta.

En este sentido, en la última década una parte importante de investigadores, académicos y profesionales del campo del marketing, han orientado sus trabajos en la investigación de las motivaciones efectividad que tiene la innovación abierta en las empresas (Noh, 2015). Los estudios iniciales se enfocaron en el análisis de estudios de casos acerca del éxito de las empresas que habían adoptado e implementado la innovación abierta, y comúnmente realizaron un análisis descriptivo del caso (Chesbrough y Crowther, 2006; Huston y Sakkab, 2006). Estudios más recientes sobre la innovación abierta publicados en la literatura del marketing, se han orientado prácticamente en elementos específicos de la industria, tales como por ejemplo la innovación abierta en los servicios financieros (Fasnacht, 2009), en el consumo de electrónicos (Christensen et al, 2005) y en la industria automotriz (Ili et al, 2010).

De igual manera, la efectividad de la innovación abierta puede generar más y mejores resultados si depende de la estrategia empresarial de las organizaciones, más que en las tendencias industriales (Keupp y Gassmann, 2009), ya que el rendimiento financiero es muy similar en las empresas de las distintas industrias (Lichtenthaler y Ernst, 2009), pero hacen falta estudios empíricos que tengan una muestra amplia de empresas para analizar los efectos que ejerce la innovación abierta en el rendimiento financiero (Noh, 2015). Por lo tanto, existe evidencia teórica y empírica que demuestra la innovación abierta es uno de los principales constructos que permiten mejorar significativamente el rendimiento financiero de las empresas (Noh, 2015), y puede ser definida como "un paradigma que asumen las empresas en la utilización tanto interna como externa de sus ideas, así como en el camino interno y externo que deberán seguir para competir en el mercado, lo cual les permitirá avanzar en su desarrollo tecnológico" (Chesbrough, 2003b: 24).

De acuerdo con esta definición, las empresas pueden aprovechar al máximo la inversión realizada en 
investigación y desarrollo, a través de la conexión de las diversas fuentes de conocimiento internas y externas a la organización (Schumpeter, 1942), lo cual permitirá que las empresas, sobre todo las pequeñas, no solamente tengan una mayor explotación externa de sus recursos de innovación (Laursen y Salter, 2006), sino también obtener una mayor cantidad de beneficios derivados del conocimiento existente al interior de las empresas (Henkel, 2006). Además, las empresas tienen la posibilidad de promover el desarrollo de los procesos de sus nuevos productos a través de la adquisición de tecnología externa, y pueden mejorar significativamente sus utilidades en el corto plazo si tomaran la decisión de licenciar su propiedad intelectual (Dahlander y Gann, 2010).

Asimismo, los procesos de la innovación abierta pueden ocurrir en dos direcciones básicas: de salida y de entrada, siendo las actividades típicas de entrada las adquisiciones y el abastecimiento de las materias primas $y / 0$ mercancías, mientras que las actividades que conllevan las de salida se incluyen las ventas y la divulgación (Dahlander y Gann, 2010). Por ello, la innovación abierta en ambas direcciones puede contribuir a mejorar potencialmente el rendimiento financiero de las empresas (Noh, 2015), siendo Procter \& Gamble un caso típico de las actividades de salida de la innovación abierta, al ser una empresa ampliamente conocida por la utilización efectiva de su conocimiento y liderazgo mundial en el desarrollo de nuevos productos (Huston y Sakkab, 2006). Además, las empresas pueden aprovechar al máximo los beneficios potenciales del conocimiento externo a través de las ventas, licenciamiento y alianzas con otras empresas (outbound open innovation) (Lichtenthaler, 2007).
Además, los efectos directos e indirectos de las actividades de la innovación abierta en el rendimiento financiero de las empresas se han incrementado significativamente, de los cuales varios estudios se han relacionado con el rendimiento innovador de las empresas, incluidos la efectividad de los costos de la investigación y el desarrollo (Caloghirou et al, 2004), la producción de patentes (Rothaermel y Alexandre, 2009) y la proporción de nuevos productos (Atuahene-Gima, 2005; Laursen y Salter, 2006; Grimpe y Sofka, 2009). Aunado a lo anterior, algunos investigadores han considerado que las actividades de la innovación abierta pueden incrementar significativamente el rendimiento innovador de las empresas (Pisano, 1990; Miotti y Sachwald, 2003), mientras que otros han sugerido que una excesiva dependencia del conocimiento externo, puede reducir los beneficios de innovación de las empresas (Ocasio, 1997; Koput, 1997; Laursen y Salter, 2006).

Así, Laursen y Salter (2006) fueron los pioneros en la utilización de métodos cuantitativos para analizar la relación existente entre la innovación abierta y el rendimiento financiero de las empresas, cuando prácticamente dominaban los estudios cualitativos en al análisis de estos dos constructos (Zhang et al, 2018). Por su parte, Koellinger (2008) consideró que las actividades de la innovación abierta no necesariamente tienen que generar un incremento significativo en el rendimiento financiero, o bien un aumento del nivel de utilidades o ganancias de las empresas. Para tratar de contrarrestar esta afirmación, Rosenbusch et al. (2011) realizan un estudio exhaustivo de las distintas actividades de la innovación abierta, aplicando un meta-análisis riguroso a 
una muestra de 21,270 empresas en la publicación de 42 diferentes estudios.

Este importante proyecto tuvo como punto de partida el tratar de dar respuesta a la pregunta sobre si las actividades de la innovación abierta tienen efectos positivos o negativos en el rendimiento financiero de las empresas, así como generar aquellos elementos que los investigadores, académicos y profesionales del campo del marketing deberán considerar para mejorar la innovación (Rosenbusch et al, 2011). Estos investigadores encontraron en su estudio diversos factores esenciales que afectan directamente a las actividades de la innovación abierta en el rendimiento financiero de las empresas, siendo algunos de ellos, por ejemplo, la edad de las empresas, los tipos de innovación que tienen las empresas y la cultura organizacional de las empresas (Rosenbusch et al, 2011).

De igual manera, desde una perspectiva del intercambio entre los beneficios y los costos asociados a las actividades de la innovación abierta, generalmente es aceptable que el grado de innovación abierta puede generar un valor marginal, más allá de si las actividades de la innovación abierta tienen un impacto positivo o negativo en el rendimiento financiero de las empresas (Zhang et al, 2018). Así, uno de los costos no monetarios en los cuales incurren comúnmente las empresas en el proceso de investigación, evaluación y filtrado de la compra de la tecnología externa, es el relacionado a la asimilación e integración al interior de la organización de la tecnología adquirida, de tal manera que esta tecnología les permita transformar y comercializar los productos generados por las empresas en el mercado en el cual participan (Tsai y Wang, 2008; West y Bogers, 2014).
Hasta entonces, el valor generado por las diversas actividades de la innovación abierta es absorbido por las empresas, y sólo serán capaces de generar un incremento significativo en el nivel de las utilidades o beneficios, en la medida que se incrementen los resultados financieros de la innovación abierta (Zhang et al, 2018). Por lo tanto, hasta antes de esta etapa de apertura las empresas eran capaces de aprovechar el conocimiento externo para su comercialización, y generar cierto nivel de utilidades o ganancias, sin embargo, al adquirir mejor tecnología las empresas tienen mayores complicaciones tanto para la integración del conocimiento externo, como para mejorar los proceso de transformación, lo cual pudiera incrementar los costos de la innovación abierta y, al mismo tiempo, reducir los beneficios que genera la innovación abierta (Koput, 1997; Laursen y Salter, 2006).

Bajo este contexto, las evidencias empíricas aportadas por los estudios publicados en la literatura del campo del marketing, que relacionan la innovación abierta y el rendimiento financiero tienen resultados encontrados, por un lado, algunos investigadores y académicos han encontrado una relación negativa entre ambos constructos $y$, por otro lado, otros autores más han encontrado una relación positiva y significativa (Caputo et al, 2016). En relación a los efectos negativos, Faems et al. (2010) argumentaron que la diversidad de las alianzas en tecnología afecta negativamente no solamente los costos del personal de las empresas, sino también la adición de valor y la ganancia marginal. Resultados similares fueron encontrados por Lazzarotti et al. (2010), quienes demostraron que las actividades de la innovación abierta 
pueden influenciar negativamente todas las forman del rendimiento empresarial de las organizaciones.

Adicionalmente, otros estudios publicados en la actual literatura del marketing no han encontrado una dirección muy clara, de la relación existente entre la innovación abierta y el rendimiento financiero de las empresas, llegando a conclusiones divergentes (Caputo et al, 2016). Por ejemplo, Hwang y Lee (2010) encontraron que las actividades de la innovación abierta generan una relación inversa con la productividad de las empresas, calculada la productividad como el total de las ventas de las empresas dividido entre el número total de los trabajadores. Asimismo, el uso moderado de los recursos del conocimiento externo incrementa significativamente el nivel de la productividad de las empresas, pero solamente hasta un número limitado de recursos externos la innovación abierta tiene importancia, en la medida que se incrementa el uso de los recursos externos disminuye el rendimiento financiero (Hwang y Lee, 2010).

Sin embargo, la mayoría de los estudios empíricos publicados han demostrado que es muy beneficioso para las empresas la adopción e implementación de las actividades de la innovación abierta en términos de retorno de las ventas (Lichtenthaler, 2009), el crecimiento en las ventas (Hung y Chiang, 2010; Chaston y Scott, 2012), aumento del número de empleados (Teirlinck y Poelmans, 2012) e incremento significativo del rendimiento financiero (Caputo et al, 2016; Zhang et al, 2018). Además, Noh (2015) analizó detalladamente el rendimiento financiero a largo plazo de las empresas que habían implementado las actividades de innovación abierta como una estrategia empresarial, encontrando que la innovación abierta no solamente afectaba las utilidades o ganancias de las empresas, sino también que mejoró sustancialmente los procesos de producción y los beneficios del mercado.

Por su parte, Huizingh (2011) encontró que las actividades de la innovación abierta tienen efectos positivos significativos en el rendimiento financiero de las empresas. Resultados similares fueron encontrados por Ahn et al. (2013), quienes llegaron a la conclusión de que las actividades de la innovación abierta están asociadas positivamente tanto con las ventas de las empresas como con el rendimiento financiero. Finalmente, Du et al. (2014) encontraron que la innovación abierta genera elevados retornos financieros en las empresas. Por lo tanto, considerando la información anteriormente presentada es posible plantear la siguiente hipótesis de investigación.

\section{H1: A mayor nivel de innovación abierta, mayor nivel de rendimiento financiero}

Además, para dar respuesta a la hipótesis planteada en este trabajo de investigación, se consideró pertinente la realización de un estudio empírico en las empresas manufactureras del Estado de Aguascalientes (México), utilizando como marco de referencia el directorio empresarial del Sistema de Información Empresarial de México para el Estado de Aguascalientes del 2018, el cual tenía registrados a finales del mes de septiembre 1,527 empresas de más de cinco trabajadores. Además, se diseñó un instrumento de recolección de la información que fue aplicado a los gerentes de las empresas manufactureras, a través de una entrevista personal a una muestra de 
300 empresas seleccionadas mediante un muestreo aleatorio simple, con un error máximo del $\pm 5 \%$ y un nivel de confiabilidad del $95 \%$, el cual se aplicó entre los meses de octubre a diciembre de 2018.

Asimismo, para la medición de las actividades de la innovación abierta se utilizó la escala desarrollada por Van de Vrande et al. (2009), quien consideró que la innovación abierta puede ser medida por medio de 7 ítems. Además, para medir el rendimiento financiero se utilizó la escala desarrollada por Tan y Litschert (1994), la cual establece que el rendimiento financiero se puede medir a través de una escala de 3 ítems. Todos los ítems de las escalas de la innovación abierta y el rendimiento financiero fueron medidos a través de una escala tipo Likert de 5 puntos con 1 = completamente en desacuerdo a $5=$ completamente de acuerdo como límites.

De igual manera, se aplicó un Análisis Factorial Confirmatorio (AFC), para la evaluación de la fiabilidad y validez de las escalas de la innovación abierta y el rendimiento financiero, utilizando para ello el método de máxima verosimilitud con el software EQS 6.2 (Bentler, 2005; Brown, 2006; Byrne,
2006). Así, la fiabilidad se midió a través del alfa de Cronbach y del Índice de Fiabilidad Compuesta (IFC) (Bagozzi y Yi, 1988). Los resultados obtenidos del AFC se presentan en la Tabla 1 y muestran que el modelo teórico analizado tiene un buen ajuste de los datos (S-BX ${ }^{2}$ (df = 31) = 97.9743; $\mathrm{p}<0.005 ; \mathrm{NFI}=0.951$; $\mathrm{NNFI}=0.950 ; \mathrm{CFI}=0.966$; RMSEA = $0.079)$, los valores del alfa de Cronbach y del IFC son superiores a 0.7 , lo cual indica la existencia de fiabilidad en las escalas del valor de marca e innovación de servicios (Nunally y Bernstein, 1994; Hair et al, 1995).

Además, los resultados del AFC indican que la totalidad de los ítems de los factores relacionados son significativos $(p<0.01)$, el valor de la totalidad de las cargas factoriales estandarizadas son superiores a 0.6 (Bagozzi y Yi, 1988), y el Índice de la Varianza Extraída (IVE) de cada par de constructos del modelo teórico de la innovación abierta y el rendimiento financiero tienen un valor superior a 0.5 (Fornell y Larcker, 1981), lo cual indica que el modelo teórico tienen un excelente ajuste de los datos, evidenciando con ello la existencia de la validez convergente.

\section{Tabla 1}

Consistencia interna y validez convergente del modelo teórico

\begin{tabular}{|c|c|c|c|c|c|c|}
\hline Variable & Indicador & Carga Factorial & Valor T Robusto & $\begin{array}{l}\text { Alfa de } \\
\text { Cronbach }\end{array}$ & IFC & IVE \\
\hline $\begin{array}{l}\text { Innovación Abierta } \\
\text { de Salida } \\
\text { (F1) }\end{array}$ & $\begin{array}{l}\mathrm{IA} 1 \\
\mathrm{IA} 2\end{array}$ & $\begin{array}{l}0.869^{* * *} \\
0.979^{* * *}\end{array}$ & $\begin{array}{l}1.000^{\mathrm{a}} \\
24.980\end{array}$ & 0.918 & 0.923 & 0.857 \\
\hline
\end{tabular}




\section{Cont... Tabla 1}

\begin{tabular}{|c|c|c|c|c|c|c|}
\hline & IA3 & $0.690^{\star \star \star *}$ & $1.000^{\mathrm{a}}$ & \multirow{6}{*}{0.908} & \multirow{6}{*}{0.910} & \multirow{5}{*}{0.670} \\
\hline \multirow{4}{*}{$\begin{array}{c}\text { Innovación Abierta } \\
\text { de Entrada } \\
\text { (F2) }\end{array}$} & IA4 & $0.755^{\star \star \star}$ & 13.925 & & & \\
\hline & & & & & & \\
\hline & IA5 & $0.823^{\star \star \star}$ & 15.858 & & & \\
\hline & IA6 & $0.909^{\star \star *}$ & 15.040 & & & \\
\hline \multirow{4}{*}{ Innovación Abierta } & IA7 & $0.895^{\star \star \star}$ & 13.837 & & & \multirow{4}{*}{0.622} \\
\hline & F1 & $0.977^{\star * *}$ & 24.980 & \multirow{3}{*}{0.832} & \multirow{3}{*}{0.752} & \\
\hline & & & & & & \\
\hline & F2 & $0.537^{\star \star \star}$ & 14.665 & & & \\
\hline \multirow{3}{*}{$\begin{array}{l}\text { Rendimiento } \\
\text { Financiero }\end{array}$} & RE1 & $0.892^{\star \star *}$ & $1.000^{a}$ & \multirow{3}{*}{0.913} & \multirow{3}{*}{0.913} & \multirow{3}{*}{0.779} \\
\hline & RE2 & $0.840^{\star \star \star}$ & 21.824 & & & \\
\hline & RE3 & $0.914^{\star \star \star}$ & 26.497 & & & \\
\hline \multicolumn{7}{|c|}{$\mathrm{S}-\mathrm{BX}^{2}(\mathrm{df}=31)=97.9743 ; \mathrm{p}<0.005 ; \mathrm{NFI}=0.951 ; \mathrm{NNFI}=0.950 ; \mathrm{CFI}=0.966 ; \mathrm{RMSEA}=0.079$} \\
\hline \multicolumn{7}{|c|}{$\begin{array}{l}\text { a = Parámetros restringidos a tal valor en el proceso de identificación. } \\
{ }_{\star \star \star} p<0.001\end{array}$} \\
\hline
\end{tabular}

\section{Fuente: elaboración propia}

Adicionalmente, la validez de correlación tiene el valor de 1. En discriminante del modelo teórico de segundo lugar, se presenta el test de la innovación abierta y el rendimiento financiero fue medida por medio de dos test, los cuales se presentan en la Tabla 2. En primer lugar, se presenta el test del intervalo de confianza (Anderson y Gerbing, 1988), el cual establece que con un intervalo de confianza del 95\%, ninguno de los elementos individuales de los factores latentes de la matriz la varianza extraída (Fornell y Larcker, 1981), el cual establece que la varianza extraída de cada par de constructos es inferior que su correspondiente IVE. Por lo tanto, de acuerdo a los resultados obtenidos de la aplicación de ambos test, es posible concluir que ambos test demuestran suficiente evidencia de la existencia de validez discriminante.

\section{Tabla 2}

\section{Validez Discriminante del modelo teórico}

\begin{tabular}{ccc}
\hline Variables & Innovación Abierta & Rendimiento Financiero \\
\hline Innovación Abierta & $\mathbf{0 . 6 2 2}$ & 0.061 \\
\hline Rendimiento Financiero & $0.095-0.589$ & $\mathbf{0 . 7 7 9}$ \\
\hline
\end{tabular}

Fuente: elaboración propia 
La diagonal representa el índice de varianza extraída (EVI), mientras que por encima de la diagonal se presenta la varianza (correlación al cuadrado). Debajo de la diagonal, la correlación estimada de los factores se presenta con un intervalo de confianza del $95 \%$.

\section{Innovación abierta y rendimiento financiero: análisis de resultados y discusión}

Para responder a la hipótesis planteada en este estudio empírico se aplicó un modelo de ecuaciones estructurales con el software EQS 6.2
(Bentler, 2005; Byrne, 2006; Brown, 2006). Además, la validez nomológica del modelo teórico de la innovación abierta y el rendimiento financiero fue analizada por medio del test de la Chicuadrada, en la cual se compararon los resultados obtenidos entre el modelo teórico y el modelo de medida, obteniendo resultados no significativos lo cual permite establecer una explicación de las relaciones observadas entre los constructos latentes (Anderson y Gerbing, 1988; Hatcher, 1994). La Tabla 3 muestra con mayor detalle los resultados obtenidos de la aplicación del modelo de ecuaciones estructurales.

\section{Tabla 3}

\section{Resultados del modelo de ecuación estructural de Segundo orden}

\begin{tabular}{cccc}
\hline Hipótesis & $\begin{array}{c}\text { Relación } \\
\text { Estructural }\end{array}$ & $\begin{array}{c}\text { Coeficiente } \\
\text { Estandarizado }\end{array}$ & $\begin{array}{c}\text { Valor T } \\
\text { Robusto }\end{array}$ \\
\hline $\begin{array}{l}\text { H1: A mayor nivel de innovación } \\
\text { abierta, mayor nivel de rendi- } \\
\text { miento financiero. }\end{array}$ & $\begin{array}{c}\mathrm{IA} \rightarrow \text { Rendi- } \\
\text { miento Finan- } \\
\text { ciero }\end{array}$ & $0.165^{\star \star *}$ & 4.571 \\
\hline $\begin{array}{l}\mathrm{S}-\mathrm{BX}(\mathrm{df}=28)=62.2861 ; \mathrm{p} \\
<0.005 ; \mathrm{NFI}=0.969 ; \mathrm{NNFI}= \\
0.972 ; \mathrm{CFI}=0.982 ; \mathrm{RMSEA}= \\
0.064\end{array}$ & & \\
\hline & & \\
\hline
\end{tabular}

Fuente: elaboración propia

La Tabla 3 muestra los resultados obtenidos de la aplicación del modelo de ecuaciones estructurales, y es posible observar que con respecto a la hipótesis $\mathbf{H}_{1}$, los resultados obtenidos $(\beta=0.165, p$ $<0.01$ ) indican que la innovación abierta tiene efectos positivos significativos en el rendimiento financiero de las empresas manufactureras, por lo cual es posible establecer que la adopción e implementación de las actividades de la innovación abierta que realicen las empresas manufactureras, afectará de manera positiva y significativa el rendimiento financiero de dichas organizaciones.

Asimismo, estos resultados tienen diversas implicaciones que son importantes determinar, siendo una primera de ellas la relacionada a que la 
generalmente un elevado porcentaje de empresas de México, al igual que en la mayoría de los países de América Latina, realizan actividades de innovación tradicional (cambios o mejoras a los productos o servicios existentes). Sin embargo, para competir en un mercado muy competitivo y globalizado como el actual, no basta con realizar actividades de innovación tradicional, sino que es sustancial la incorporación de prácticas de innovación abierta de entrada y salida, de tal manera que permita a las empresas no solamente incrementar sus actividades de innovación sino también su nivel de rendimiento financiero.

Adicionalmente, la innovación abierta requiere que las empresas manufactureras realicen cambios importantes al interior y exterior de la organización, de tal manera que les permita colaborar con otras empresas, compartir conocimientos, experiencias y formas distintas de hacer las cosas, con el objetivo de enriquecer y mejorar los procesos de la innovación y, a su vez, lograr tanto un mayor valor añadido a los productos como incrementar el nivel del rendimiento empresarial. Estos resultados son similares a los obtenidos por Noh (2015), quien encontró que la innovación abierta implementada en las empresas manufactureras permitió a las organizaciones no solamente mejorar significativamente sus procesos de producción, sino también lograr un mayor nivel de rendimiento empresarial.

Una segunda implicación derivada de los resultados obtenidos es que para que las empresas obtengan un mayor nivel del rendimiento financiero, los gerentes de las empresas tienen que adoptar e implementar todas aquellas actividades que fomente la innovación abierta al interior de la organización, lo cual implica modificar los patrones generales de conducta, creencias y valores que el personal de la organización comparten, así como generar un clima de confianza que permita adquirir y compartir los conocimientos y habilidades entre los trabajadores y empleados de la empresa. En otras palabras, lo anterior significa renovar la cultura organizacional y la forma tradicional de trabajo de la empresa, dado que la innovación abierta requiere romper paradigmas en los flujos de entrada y salida deliberada de información.

En este sentido, es importante establecer la importancia que tiene la capacitación adecuada para que los empleados y trabajadores de la organización, no sólo manejen adecuadamente la información referente a las actividades de la innovación abierta, sino también para que sean competentes y productivos en el desarrollo de dichas actividades, lo cual facilitaría la optimización del rendimiento productivo en la organización, al mejorar significativamente las capacidades de los empleados y trabajadores mediante el perfeccionamiento de las destrezas, conductas y habilidades que les permitan familiarizarse más fácilmente con los procesos que conlleva la innovación abierta de entrada y salida, lo cual podría tener como resultado un mayor nivel de rendimiento empresarial.

Finalmente, una tercera implicación de los resultados obtenidos es que los gerentes de las empresas de la industria manufacturera, no deberán considerar a la innovación abierta como una simple estrategias empresarial de la organización, sino más bien como parte de las actividades cotidianas y sustanciales que se realizan día con día en la empresa, lo cual requiere que los gerentes tengan un amplio conocimiento 
tanto de los requerimientos de la adopción e implementación de la innovación abierta, como de las diversas ventajas que esta ofrece. Estos resultados son similares a los obtenidos por Du et al. (2014) quienes encontraron que las actividades de la innovación abierta de las empresas manufactureras tienen un mayor nivel de rendimiento financiero en las empresas si se involucra en ellas al personal de la organización.

\section{Conclusiones}

Las actividades de innovación abierta que realizan las empresas manufactureras tienen efectos positivos significativos en su nivel de rendimiento financiero. Las prácticas de innovación abierta tienen una estrecha relación con el rendimiento financiero de las empresas, lo cual pone de manifiesto la importancia que tiene el conocimiento derivado de la propia organización, de la colaboración directa con otras empresas y con sus principales clientes, el cual tiene como principal objetivo obtener acceso a los conocimientos derivados de la colaboración, o bien para generar sinergia entre las empresas con la finalidad de compartir conocimientos y experiencias que faciliten el desarrollo de las actividades de la innovación abierta, siendo estos resultados similares a los obtenidos por Huizingh (2011).

La mayoría de las empresas manufactureras están más orientadas en el desarrollo de las prácticas de la innovación abierta de salida que en las prácticas de entrada, lo cual significa que para este tipo de empresas son esenciales aquellas actividades que involucran las ventas de sus licencias y el ofrecimiento de regalías a otras empresas derivados de los beneficios de los derechos de su propiedad intelectual. Por lo tanto, si las empresas manufactureras pretender desarrollar más sus actividades de innovación, es imprescindible que orienten sus esfuerzos hacia las prácticas de la innovación abierta de entrada, como por ejemplo fomentar la colaboración con nuevas empresas o con empresas previamente establecidas en el mercado, con la finalidad de compartir conocimientos, estrategias y métodos para lograr sinergias empresariales que les permitan incrementar su nivel de rendimiento empresarial.

Finalmente, dado que el objetivo transcendental de las empresas manufactureras es mejorar significativamente su nivel de rendimiento financiero, es posible concluir que para lograr este objetivo las empresas deberán no solamente adoptar a las actividades de la innovación abierta, sino también fomentar las actividades de redes de colaboración externas para mejorar sus procesos de innovación, de tal manera que brinde a las organizaciones nuevas formas de innovar sus productos, para que les genere diversas ventajas competitivas que coadyuven con el retorno de la inversión en un menor plazo, y que favorezcan una mayor participación del mercado en comparación con sus principales competidores, y un incremento significativo en su nivel de rendimiento financiero.

Adicionalmente, este estudio empírico también tiene diversas limitaciones que son importantes establecer, siendo una primera de ellas la relacionada con la muestra utilizada, ya que solamente se consideraron a las pequeñas y medianas empresas del sector manufacturero que tenían registrados de 5 a 250 trabajadores, por lo cual en estudios futuros sería 
pertinente considerar en la muestra a aquellas pequeñas empresas manufactureras que cuenten con una cantidad menor de 5 trabajadores para corroborar los resultados obtenidos.

Una segunda limitación es que las escalas utilizadas para la recolección de la información, se aplicaron solo a las pequeñas y medianas empresas manufactureras del Estado de Aguascalientes (México), por lo cual en estudios futuros será necesario aplicar esta misma encuesta a las pequeñas y medianas empresas manufactureras de otros estados del país para corroborar los resultados obtenidos.

Una tercera y última limitación es que las encuestas fueron aplicadas a los gerentes $y / o$ propietarios de las pequeñas y medianas empresas manufactureras, con lo cual se asume que éstos tienen un amplio conocimiento de las actividades de innovación abierta y del desempeño financiero, por lo cual en estudios futuros sería adecuada la aplicación de las mismas encuestas tanto al personal de la empresa como a sus principales consumidores para corroborar si los resultados difieren o no de los obtenidos.

\section{Referencias bibliográficas}

Ahn, Joon, Mo; Minshall, Tim y Mortara, Letizia (2015), Open innovation: A new classification and its impact on firm performance in innovative SMEs. Journal of Innovation Management, Año 2, No. 3, pp 33-54.

Ahn, Joon, Mo; Mortara, Letizia y Minshall, Tim (2013), The effects of open innovation on firm performance: A capacity approach. STI Policy Review, Año 1, No. 4, pp 74-93.

Anderson, James y Gerbing, David (1988), Structural equation modeling in practice: a review and recommended two-step approach. Psychological Bulletin, Año 1, No. 13, Sejong, Korea, Science and Technology Policy Institute, pp 411-423.

Atuahene-Gima, Kwaku (2005), Resolving the capability: Rigidity paradox in new product innovation author(s). Journal of Product Innovation Management, Año 3, No. 23, Nueva York, Estados Unidos, Sage Publications, pp 27-47.

Bagozzi, Richard y Yi, Youjae (1988), On the evaluation of structural equation models. Journal of the Academy of Marketing Science, Año 1, No. 16, Berlin, Alemania, Springer-Verlag, pp 74-94.

Bentler, Peter (2005), EQS 6 Structural Equations Program Manual. California, Multivariate Software, pp 422.

Brown, Timothy (2006), Confirmatory Factor Analysis for Applied Research. Nueva York, The Guilford Press, pp 493.

Byrne, Barbara (2006) Structural Equation Modeling With EQS, Basic Concepts, Applications, and Programming. Londres, LEA Publishers, pp, 429.

Caloghirou, Yannis; Ioanna, Kastelli y Aggelos, Tsakanikas (2004), Internal capabilities and external knowledge sources: Complements or substitutes for innovation performance? Technovation, Año 1, No. 24, Atenas, Grecia, National Technical University of Athens, pp 29-39.

Caputo, Mauro; Lamberti, Emilia; Cammarano, Antonello y Michelino, Francesca (2016), Exploring the impact of open innovation on firm performance. Management Decision, Año 7, No. 54, Reino Unido, Emerald Group Publishing Ltd, pp 1789-1812. 
Chaston, Ian, y Gregory J. Scott (2012), Entrepreneurship and open innovation in an emerging economy. Management Decision, Año 7, No. 50, Reino Unido, Emerald Group Publishing Ltd, pp 1161-1177.

Chesbrough, Henry, William (2003a), The era of open innovation. MIT Sloan Management Review, Año 3, No. 44, Estados Unidos, Massachusetts Institute of Technology pp 35-41.

Chesbrough, Henry, William (2003b), Open Innovation. Cambridge, Massachusetts, Harvard University Press.

Chesbrough, Henry, William (2006), Open Business Models: How to Thrive in the New Innovation Landscape. Boston, Massachusetts, Harvard Business School Press.

Chesbrough, Henry, William y Adrienne, Kardon, Crowther (2006), Beyond high-tech: Early adopters of open innovation in other industries. RyD Management, Año 3, No. 36, Oxford, Reino Unido, John Wiley \& Sons, pp 229-236.

Christensen, Jens, Frøslev; Michael, Holm, Olesen y Jonas, Sorth, Kjær (2005), The industrial dynamics of open innovation: Evidence from the transformation of consumer electronics. Research Policy, Año 10, No. 34, Holanda, Elsevier B.V, pp15331549.

Cohen, Wesley y Daniel, Levinthal (1990). Absorptive capacity: A new perspective of learning and innovation. Administrative Science Quarterly, Año 1, No. 35, Estados Unidos, Sage Publications, pp 128-152.

Cooper, Robert (2008), The stage-gate idea-to-lunch process: Update, what's new and NexGen systems. Journal of Product Management,
Año 3, No. 25, Canadá, pp 213-232.

Dahlander, Linus, y Gann, David (2010), How open is innovation? Research Policy, Año 6, No. 39, Holanda, Elsevier B.V, pp 699-709.

Du, Jingshu; Leten, Bart y Vanhaverbeke, Wim (2014), Managing open innovation projects with science-based and market-based partners. Research Policy, Año 5 No. 43, Holanda, Elsevier B.V, pp 828-840.

Faems, Dries; De Visser, Matthias; Andries, Petra y Van Looy, Bart (2010), Technology alliance portfolios and financial performance: Value-enhancing and cost-increasing effects of open innovation. Journal of Product Innovation Management, Año 6, No. 27, Estados Unidos, Sage Publications, pp 785-796.

Fasnacht, Daniel (2009), Open Innovation in the Financial Services. Berlin, Heidelberg: Springer-Verlag.

Fornell, Claes y Larcker, David (1981), Evaluating structural equation models with unobservable variables and measurement error. Journal of Marketing Research, Año 1, No. 18, Chicago. United States, Cambridge University Press, pp. 39-50.

Fosfuri, Andrea (2006), The licensing dilemma: Understanding the determinants of the rate of technology licensing. Strategic Management Journal, Año 12, No. 27, Estados Unidos, John Wiley \& Sons Inc. pp 1141-1158.

Gambardella, Alfonso; Giuri, Paola y Luzzi, Alessandra (2007), The market for patents in Europe. Research Policy, Año 1, No. 36, Holanda, Elsevier B.V, pp 1163-1183.

Greco, Marco; Grimaldi, Michele y Cricelli, Livio (2015), Open innovation actions and innovation performance: 
A literature review of European empirical evidence. European Journal of Innovation Management, Año 2 No. 18, Reino Unido, Emerald Group Publishing Ltd, pp 150-171.

Grimpe, Christoph y Sofka, Wolfgang (2009), Search patterns and absorptive capacity: Low-and-high-technology sector in European countries. Research Policy, Año 3, No. 38, Dinamarca, 495-506.

Hair, Joseph, Anderson, Rolph, Tatham, Ronald y Black, William (1995). Multivariate Data Analysis with Readings. New York. Prentice-Hall, pp. 761.

Hatcher, Larry (1994). A Step-by-Step Approach to Using the SAS System for Factor Analysis and Structural Equation Modeling. North Carolina, SAS Institute Inc, pp 429.

Henkel, Joachim (2006), Selective revealing in open innovation processes: The case of embedded Linux. Research Policy, Technische Universität München, Alemania, Año 7, No. 35, pp 953-969.

Huizingh, Eelko H (2011), Open innovation: State of the art and future perspectives. Technovation, University of Groningen, Paises Bajos, Año 1, No. 31, pp 2-9.

Hung, Kuang-Peng y Chiang, Yun-Hwa (2010), Open innovation proclivity, entrepreneurial orientation and perceived firm performance. International Journal of Technology Management, Año3/4, No. 52, Taiwan, pp 257-274.

Huston, Larry y Sakkab, Nabil (2006), Connect and develop: Inside Procter y Gamble's new model for innovation. Harvard Business Review, Año 3, No. 84, , Estados Unidos de America, pp 58-66.
Hwang, Junseok y Lee, Youngjin (2010), External knowledge search, innovative performance and productivity in the Korean ICT sector. Telecommunications Policy, Año 10, No. 34, Corea del sur, pp 562-571.

Ili, Serhan; Albers, Albert y Miller, Sebastian (2010), Open innovation in the automotive industry. RyD Management, Año 3, No. 40, Alemania pp 246-255.

Keupp, Marcus Matthias y Gassmann, Oliver (2009), Determinants and archetype users of open innovation. RyD Management, Año 4, No. 39, Alemania, pp 331-341.

Koellinger, Philipp, D (2008), The relationship between, technology, innovation, and firm performance: Empirical evidence from e-business in Europe. Research Policy, Año 8, No. 37, Holanda, pp 1317-1328.

Koput, Kenneth, W (1997), A chaotic model of innovative search: Some answers, many questions. Organizational Science, Año 5, No. 8, Estados Unidos de America, pp 528-542.

Laursen, Keld y Salter, Ammon J (2006), Open for innovation: The role of openness in explaining innovation performance among UK manufacturing firms. Strategic Management Journal, Año 1, No. 27, Reino Unido, pp 131-150.

Lazzarotti, Valentina; Manzini, Raffaella y Pellegrini, Luisa (2010), Open innovation models adopted in practice: An extensive study in Italy. Measuring Business Excellence, Año 4, No. 14, Carlo Cattaneo University LIUC, Italia, pp 11-23.

Lichtenthaler, Ulrich (2007), The drivers of technology licensing: An industry comparison. California Management Review, International School of Management, Año 4, No. 49, Ale- 
mania, pp 67-89.

Lichtenthaler, Ulrich (2008), Open innovation in practice: An analysis for strategic approaches to technology transactions. IEEE Transactions on Engineering Management, International School of Management, Año 1, No. 55, Alemania, pp 148-147.

Lichtenthaler, Ulrich (2009), Outbound open innovation and its effects on firm performance: Examining environmental influences. RyD Management, Año 4. No. 39, International School of Management, Alemania, pp 317-330.

Lichtenthaler, Ulrich (2015), A note on outbound open innovation and firm performance. RyD Management, Año 5, No. 45, International School of Management, Alemania, pp 606-608.

Lichtenthaler, Ulrich y Ernst, Holger (2009), Opening up the innovation process: The role of technology aggressiveness. RyD Management, Año 1, No. 39, International School of Management, Alemania, pp 38-54.

Linstone, Harold (2010), Comment on is open innovation a field of study or a communication barrier to theory development? Technovation, Año 11/12 No. 30, Estados Unidos de America, pp 556-566.

March, James, G (1991), Exploration and exploitation in organizational learning. Organization Science, Año 1, No. 2, Estados Unidos de America, pp 71-87.

Miotti, Luis, Egidio y Sachwald, Frederique (2003), Co-operative RyD: Why and with whom? An integrated framework of analysis. Research Policy, Año 8, No. 32, Francia,pp 1481-1499.

Mowery, David, C (2009), Plus can change: Industrial RyD in the third industrial revolution. Industrial and Corporate Change, Año 1, No. 18, University of California, Estados Unidos de America, pp 1-50.

Noh, Yonghwi (2015), Financial effects of open innovation in the manufacturing industry. Management Decision, Año 7, No. 53, Myongji University, Corea del sur,pp 1527-1544.

Nunally, Jum y Bernstein, Ira (1994). Psychometric Theory. New York. McGraw-Hill. Pp.736.

Ocasio, William (1997), Towards an attention-based view of the firm. Strategy Management Journal. Año S1, No.18, Northwestern University, Estados Unidos de America, pp 187206.

Pisano, Gary, P (1990), The RyD boundaries of the firm: An empirical analysis. Administration Science Quarterly, Año 3, No. 35, Estados Unidos de America, pp 153-176.

Rosenbusch, Nina; Brinckmann, Jan y Bausch, Andreas (2011), Is innovation always beneficial? A meta-analysis of the relationship between innovation and performance in SMEs. Journal of Business Venturing, Año 4, No. 26, Alemania, pp 441-457.

Rothaermel, Frank T y Alexandre, Maria, Tereza (2009), Ambidexterity in technology sourcing: The moderating role of absorptive capacity. Organizational Science, Año 4 No. 20, Atlanta, Estados Unidos de America, pp 759-780.

Schroll, Alexander y Mild, Andreas (2012), A critical review of empirical research on open innovation adoption. Journal für Betriebswirtschaft, Año 2, No. 62, Austria, pp 85-118.

Schumpeter, Joseph A (1942), Capitalism, Socialism, and Democracy. London: Unwin University Books, 


\section{pp 1-431}

Teirlinck, Peter y Poelmans, Eline (2012), Open innovation and firm performance in small-sized RyD active companies in the chemistry industry: The case of Belgium. Journal of Business Chemistry, Año 3, No. 9, Universidad en Lovaina, Bélgica, pp 117-132.

Tsai, Kuen, Hung y Wang, Jiann Chyuan (2008), External technology acquisition and firm performance: A longitudinal study. Journal of Business Venturing, Año 1, No. 23, Taiwan, pp 91-112.

Vanhaverbeke, Wim; Duysters, Geert y Noorderhaven, Niels (2002), External technology sourcing through alliances or acquisitions: An analysis of the application-specific integrated circuits industry. Organizations Science, Año 6, No.13, Estados Unidos de America, pp 714-733.
West, Joel y Bogers, Marcel (2014), Leveraging external sources of innovation: A review of research on open innovation. Journal of Product Innovation Management, Blackwell Publishing, Año 4, No. 31, pp 814831.

West, Joel; Vanhaverbeke, Wim y Chesbrough, Henry (2006), Open innovation: A research agenda. Open Innovation: Researching a New Paradigm. Oxford: Oxford University Press.

Zhang, Si; Yang, Delin; Qiu, Shumin; Bao, Xiang y Li, Jizhen (2018), Open innovation and firm performance: Evidence from Chinese mechanical manufacturing industry. Journal of Engineering and Technology Management, Año 1, No.48, China, pp 76-86. 